\title{
Boosting the Renewable Energy Economy with NRGcoin
}

\author{
Mihail Mihaylov \\ AI lab, Vrije Universiteit Brussel \\ Brussels, Belgium \\ mmihaylo@vub.ac.be \\ Iván Razo-Zapata \\ Luxembourg Institute of Science and Technology \\ Esch-Sur-Alzette, Luxembourg \\ ivan.razo-zapata@list.lu
}

\author{
Roxana Rădulescu \\ AI lab, Vrije Universiteit Brussel \\ Brussels, Belgium \\ rradules@vub.ac.be \\ Ann Nowé \\ AI lab, Vrije Universiteit Brussel \\ Brussels, Belgium \\ ann.nowe@vub.ac.be
}

\section{ABSTRACT}

Recent work introduced the concept of NRGcoin [1] - a decentralized digital currency for renewable energy, based on Blockchain technology. In this abstract we summarize the concept and outline how this currency benefits each stakeholder in the smart grid and the renewable energy economy as a whole.

The NRGcoin concept is visualized in Figure 1. When a prosumer generates energy and injects it in the grid (1), she receives information from the grid operator (2) on the balance of supply and demand in the micro-grid (or district). This information is then used by the smart meter at that agent's home to securely create (3) (also issue, or "mint"), new digital units (or NRGcoins) for that prosumer. For every $1 \mathrm{kWh}$ of renewable energy that the prosumer injects in the grid, her smart meter creates 1 NRGcoin. At any time, the prosumer can offer to sell her coins (4) on the NRGcoin currency market - a FOREXtype currency exchange. Consumers join the NRGcoin market and buy NRGcoins (5) using fiat currency, e.g. Euro, Dollar, Pound, etc. When consumers use green energy from the grid (6), their smart meter automatically pays for that consumption using NRGcoins (7), instead of traditional currency. $1 \mathrm{kWh}$ green energy consumption from the grid costs 1 NRGcoin, regardless of the current retail cost of energy. Nevertheless, since NRGcoin is linked to energy, which in turn is linked to money, NRGcoin is indirectly linked to the monetary value of energy, unlike other digital currencies, such as Bitcoin, which are not linked to any particular value. Thus it is expected that NRGcoin will always fluctuate closely around the retail value of electricity. All digital transactions of NRGcoins, including their creation, are governed by the decentralized NRGcoin protocol - a piece of software running on the peer-to-peer network of smart meters. This protocol is based on the Blockchain technology [2].

Thus the NRGcoin concept offers a number of benefits to each stakeholder in the smart grid. It serves as:

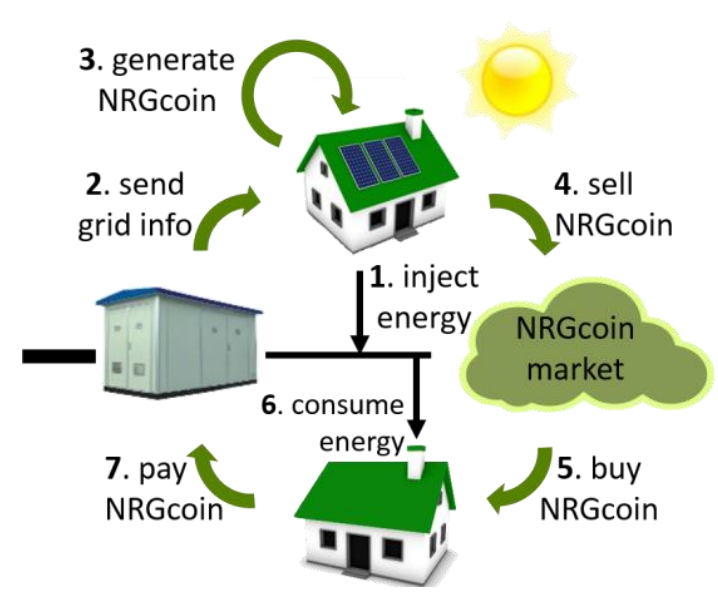

Figure 1. The NRGcoin concept

- a subsidy scheme for renewable installations with lower risk against policy change for prosumers. The issuance of the currency is governed by a decentralized protocol that can only be changed by a majority vote and thus not by individual market actors.

- a currency that offers consumers an alternative payment choice and cheaper green energy. High NRGcoin supply on the currency exchange market will drive NRGcoin price down, resulting in lower cost of green energy.

- a financial tool offering faster cash flow and profit to retailers and grid operators. As a peer-to-peer currency, NRGcoin can process micro-payments, e.g. payments for energy every 15 minutes, at virtually zero overhead costs.

- a mechanism that reduces the need for government support schemes, since prosumers are generating their own money, thus saving on budget.

- $\quad$ an asset from which investors profit when electricity prices increase. Buying the currency at the current retail price of energy and selling at a later point will generate profits, as 
it has been estimated that electricity prices tend to increase, rather than decrease [4].

Since NRGcoins are generated (or spent) by supplying (or consuming) renewable energy to (from) the grid, the currency allows individuals to express their valuation of green energy by placing corresponding bids on the market. For example, some consumers may be willing to pay for renewable energy a higher price than the retail cost of energy, thus contributing directly to the growth of the renewable sector.

Given the above advantages to stakeholders, the NRGcoin concept has the long-term potential to boost the renewable energy economy in a way similar to how community currencies boost local economies [3]. For example, a PV panel manufacturer may offer discount for their panels if paid in NRGcoin. In doing so, the manufacturer obtains the coins at a rate cheaper than that offered at the currency market, while the consumer obtains cheaper panels. These price reductions benefit each party and increases the velocity of the currency and hence its value, which in turn increases the incentives to install renewables.

\section{ACKNOWLEDGMENT}

This research has been funded by the European Union's Seventh Programme for research, technological development and demonstration under the grant agreement number 324321, project SCANERGY, and by Flanders Innovation \& Entrepreneurship, SBO project 140047 Smile-IT.

\section{REFERENCES}

[1] M. Mihaylov, S. Jurado, K. Van Moffaert, N. Avellana, and A. Nowe, "NRG-X-Change: a Novel Mechanism for Trading of Renewable Energy in Smart Grids," in Proceedings of the 3rd International Conference on Smart Grids and Green IT Systems, pp. 101-106, Apr. 2014

[2] S. Nakamoto, "Bitcoin: A peer-to-peer electronic cash system", https://bitcoin.org/bitcoin.pdf, 2008.

[3] G. Seyfang. Community currencies: small change for a green economy. Environment and planning, 33(6):975-996, 2001.

[4] P.V. Parity, Electricity Prices Scenarios until at Least the Year 2020 in Selected EU Countries, München, 2012. 